\title{
MHC genes in Invertebrates: The Echinodermata
}

\author{
Michel Leclerc $^{1 *}$, Ariane Jolly ${ }^{2}$ and Pierre de la Grange ${ }^{2}$ \\ 1556 rue Isabelle Romée, 45640 Sandillon, France \\ ${ }^{2}$ Genosplice, Paris, France
}

*Corresponding author: Michel Leclerc, 1556 rue Isabelle Romée, 45640 Sandillon, France, Tel: 0238410209; E-mail: mleclerc45@gmail.com

\begin{abstract}
The MHC is a set of genes that codes for cell surface proteins essential for the acquired immune system to recognize foreign molecules in vertebrates and MHC II gene was described in Echinodermata for the first time. For the present time, MHC Class I gene was not found in a significant manner so for concluding the existence of MHC I gene in Echinodermata, further studies will be necessarily done.
\end{abstract}

Keywords: Echinodermata; Invertebrates; MHC Genes

Received Date: May 31, 2019; Accepted Date: June 7, 2019; Published Date: June 14, 2019

\section{Introduction}

As Janeway CA [1] wrote in 2001, "The function of MHC molecules is to bind peptide fragments derived from pathogens and display them on the cell surface for recognition by the appropriate $\mathrm{T}$ cells. The consequences are almost always deleterious to the pathogen-virus-infected cells are killed, macrophages are activated to kill bacteria living in their intracellular vesicles, and B cells are activated to produce antibodies that eliminate or neutralize extracellular pathogens. Thus, there is strong selective pressure in favor of any pathogen that has mutated in such a way that it escapes presentation by an MHC molecule. Two separate properties of the MHC make it difficult for pathogens to evade immune responses in this way. First, the MHC is polygenic: it contains several different MHC class I and MHC class II genes, so that every individual possesses a set of MHC molecules with different ranges of peptide-binding specificities. Second, the MHC is highly polymorphic; that is, there are multiple variants of each gene within the population as a whole. The MHC genes are, in fact, the most polymorphic genes known.

Because of the polygeny of the MHC, every person will express at least three different antigen-presenting MHC class I molecules and three (or sometimes four) MHC class II molecules on his or her cells. In fact, the number of different MHC molecules expressed on the cells of most people is greater because of the extreme polymorphism of the MHC and the codominant expression of MHC gene products.

Citation: Michel Leclerc, MHC genes in Invertebrates: The Echinodermata. J Clin Cases Rep 2(4): 102-105. DOI: https://doi.org/10.46619/joccr.2019.2-1049 
http://www.tridhascholars.org | October-2019

The term polymorphism comes from the Greek poly, meaning many, and morphe, meaning shape or structure. As used here, it means within-species variation at a gene locus, and thus in its protein product; the variant genes that can occupy the locus are termed alleles. There are more than 200 alleles of some human MHC class I and class II genes, each allele being present at a relatively high frequency in the population. So, there is only a small chance that the corresponding MHC locus on both the homologous chromosomes of an individual will have the same allele; most individuals will be heterozygous at MHC loci. The particular combination of MHC alleles found on a single chromosome is known as an MHC haplotype. Expression of MHC alleles is codominant, with the protein products of both the alleles at a locus being expressed in the cell, and both gene products being able to present antigens to $\mathrm{T}$ cells. The extensive polymorphism at each locus thus has the potential to double the number of different MHC molecules expressed in an individual and thereby increases the diversity already available through polygeny.

In addition to the highly polymorphic 'classical' MHC class I and class II genes, there are many genes encoding MHC class I-type molecules that show little polymorphism; most of these have yet to be assigned a function. They are linked to the class I region of the MHC and their exact number varies greatly between species and even between members of the same species. These genes have been termed MHC class IB genes; like MHC class I genes, they encode $\beta 2$-microglobulin-associated cellsurface molecules. Their expression on cells is variable, both in the amount expressed at the cell surface and in the tissue distribution".

In human, the main function of major histocompatibility complex (MHC) Class II molecules, is to present processed antigens which are derived primarily, from exogeneous sources.

Constitutive expression of MHC Class II molecules, is also confined to professional antigen- presenting cells (APC) of the immune system [2].

Since, we have discovered the IPA (Invertebrate Primitive Antibody) [3-6], to acquire a better understanding of the invertebrate immune system, it seemed useful to look for MHC genes (HLA-DRB1 gene) (HLA-C gene) in invertebrates with Ophiocomina nigra (Ophuirids), Antedon bifida (Crinoïds) as model of studies. On the other hand we'll have a look on a MHC Class I gene (HLA-C gene) corresponding to a molecule which is a heterodimer consisted of a heavy chain and a light one (beta-2 microglobulin).

\section{Materials and Methods}

Animals: Ophiocomina nigra (Ophuirid) Antedon bifida (Crinoïd) were obtained at the station of Biologie Marine of Roscoff, France. Obtention of ophuirid and crinoïd mRNA: Digestive coeca were excised from their bodies and mRNA was obtained from Uptizol (Interchim) then quality controls were operated [7].

Sequencing: Sequencing was made on Illumina Next Seq 500 with paired-end: 2.75 bp. Transcriptome was assembled from RNA-Seq fastq files using Trinity v2.1.1 [8] with default parameters. A BLAST database was created with the assembled transcripts using make blast $\mathrm{dB}$ application from ncbi-blast+ (v2.2.31+). The sequences of transcripts of interest were then blasted against this database using blastn application from ncbi-blast+ [9] with parameter word_size 7. 
http://www.tridhascholars.org | October-2019

\section{Results}

MHC gene Class II appears in the genome of Ophiocomina nigra and Antedon bifida one, in a significant manner. The transcriptomes are given in the following tables, with the sequences just after. First, Ophiocomina nigra results show the"HLA-DRB1" transcriptome which possesses a short sequence but a specific one.

\begin{tabular}{|c|c|c|c|c|c|c|c|c|c|}
\hline Query ID & Quer Name & SubjectID & Identity (\%) & Length & Mismatch & Gap open & Query cover (\%) & E-value & Bitscore \\
\hline NM_002124.3 & HLA-DRB1 & $\begin{array}{l}\text { TRINITY_DN4807_c1 } \\
\text { g1_i1 }\end{array}$ & 90,77 & 65,00 & 3 & 2 & 5,00 & $1,00 \mathrm{E}-15$ & 84,20 \\
\hline
\end{tabular}

>TRINITY_DN4807_c1_g1_i1

5'CATATAGTTTAGGGGGTTATAAAAAAATGACTCCGGTTACTGACATATTTGGGACCCCAA

CTGTCCAAAGAAAATTATAGCCCCTATAAATTATAATTTATTAATTTTTGTTTTCTCTTG

TATAGGGACCAGAGCCAATCCCACTGGAAGTTAGGGCACGAGCAGTCAAAGACCAATTTT

AAATGTAAAAAAAAAAAAAAAAAAAAATAAAAAATTAAAAAAAAAAAAAAAAAAATAAAA

AATTAAAAAAAAAAAAAAAAAAATAAA3'

Secondly with $A$ bifida

We find the transcriptome called HLA-DRB1 Antedon bifida with the following e-value, identity, bitscore

\begin{tabular}{|c|c|c|c|c|c|c|c|c|c|}
\hline QueryID & Query Name & SubjectID & Identity (\%) & Length & Mismatch & Gap open & Query cover (\%) & E-value & Bitscore \\
\hline NM_002124.3 & HLA-DRB1 & $\begin{array}{l}\text { TRINITY_DN2 } \\
\text { 0232_c5_g3_i1 }\end{array}$ & 83,05 & 59 & 9 & 1 & 5,00 & $4,00 \mathrm{E}-06$ & 52,80 \\
\hline
\end{tabular}

The sequence in 5'-3' shows also a short one as for the ophuirid: Ophiocomina nigra but remains higly specific as following:

>TRINITY_DN20232_c5_g3_i1

5'GCATGCCTGTAATCCCAGCTACTTGGGAGGCTGAGGCAGGAGAATCACTTGAACCCAGGA

GGCAGAGGTTGTGGTGAGCCGAGATCATGCCATCGCACTCCAGCCTGGGCAATAAGAGCG

AAACTCCGGTCTCAAACAAACAAACAAAAAACAAAACAAAAACAAACAAAAAACAAAACA

AAAACAAACAAAAAACAAAACAAA3'

MHC Class I gene was not found, at least in a significant manner (the e-value was not significative).

\section{Conclusion}

It is obvious that MHC class II gene (HLA-DRB1 gene) exist in Echinodermata, at least in Echinodermata which possess a sophisticated immune system as Asterids, Ophuirids, Crinoïds. It would be interesting also to study MHC system in Echinodermata which present only innate immune response as Echinids, Holothurids [10].

The HLA-DRB1 gene is a part of a family of genes called the human leukocyte antigen (HLA) complex.

The protein encoded by this gene belongs to the HLA class II beta chain paralogues. The class II molecule is a heterodimer consisting of an alpha (DRA) and a beta chain (DRB), both anchored in the membrane. It plays a central role in the immune system by presenting peptides derived from extracellular proteins to T helper cells. Class II molecules are constitutively expressed in professional antigen presenting cells (APC: B lymphocytes, dendritic cells, macrophages) and could be induced in non-professional APCs. DRB1 is expressed at a level five times higher than its paralogues DRB3, DRB4 and DRB5. DRB1 is present in all individuals. Allelic variants of DRB1 are linked with either none or one of the genes DRB3, DRB4 and DRB5. There are 4 related pseudogenes: DRB2, DRB6, DRB7, DRB8 and DRB9. 
http://www.tridhascholars.org| October-2019

We try now to look for MHC class I genes in Ophuirids and Crinoïds.

The HLA-C gene (we looked for) belongs also to the HLA complex: it helps the immune system to distinguish the body own proteins from proteins made by foreign invaders such as viruses and bacteria [11-13].

Although all efforts to find in Invertebrates a Major Histocompatibility Complex System have been unsuccessful, we find today, for the first time, MHC class II gene (HLA-DRB1 gene) in Echinodermata: It's of great novelty. It is a fundamental discovery in the domain of Comparative Immunology.

\section{References}

1. Janeway CA, Travers P, Walport M, et al. (2001) Immunobiology ( $5^{\text {th }}$ Edn.) New York: Garland Sciences.

2. Holling TM, Schooten E, van Den Elsen PJ (2004) Function and regulation of MHC class II molecules in T lymphocytes: of mice and men. Human Immunology 65(4): 282-290.

3. Leclerc M (2018) The complement system in Echinodermata includes the Lectin Pathway. Archives of Immunology and Allergy 1(2): 1-2.

4. Leclerc M (2016) Evidence of Fab gene in an Invertebrate: Ophiocomina nigra (Echinodermata). EC Microbiology 3(5): 539-541.

5. Leclerc M (2016) Evidence of Fc receptor gene in an Invertebrate: Ophiocomina nigra (Echinodermata). International Journal of Biotechnology and Bioengineering 4(3): 35-36.

6. Leclerc M (2018) Evidence of Immune Genes in the Crinoid: Antedon Bifida Evidence of A. Bifida Igkappa Gene, Fc Receptor Gene. International Journal of Vaccine Research 3(1): 1-2.

7. Vincent N, Osteras M, Otten P, et al. (2014) A new gene in A Rubens: A sea star IGKappa gene. Meta Gene 2: 320-322.

8. Grabherr MG, Haas BJ, Yassour M, et al. (2011) Full-length transcriptome assembly from RNA-seq data without a reference genome. Nature Biotechnology 29(7): 644-652.

9. Altschul SF, Gish W, Miller W, et al. (1990) Basic local Alignment search tool. Journal of Molecular Biology 215(3): 403-410.

10. Leclerc M (2018) Evidence of complement genes in the crinoid: Antedon Bifida. Comparisons with other Echinodermata. International Journal of Biotechnology and Bioengineering 5(1): 117-118.

11. Hiby SE, Walker JJ, O'shaughnessy KM, et al. (2004) Combinations of material KIR and fetal HLA-C genes influence the risk of preeclampsis and reproductive success. The Journal of Experimental Medicine 200(8): 957-965.

12. Khakoo SI, Thio CL, Martin MP, et al. (2004) HLA and NK cell inhibitory receptor genes in resolving hepatitis C virus infection. Science 305: 872-874.

13. Nair RP, Stuart PE, Nistor I, et al (2006) Sequence and haplotype analysis supports HLA-C as the psoriasis susceptibility 1 gene. AJHG 78(5): 827-851. 